\title{
Características clínico patológicas del cáncer de mama asociadas a la expresión del marcador celular Ki-67 en el Hospital Nacional Edgardo Rebagliati Martins, 2013-2017 \\ Clinical pathological features of breast cancer associated with the expression of the Ki-67 cell marker at Edgardo Rebagliati Martis National Hospital, 2013-2017
}

\author{
Silvia Lucía Mayanga Sausa, a \\ ${ }^{1}$ Unidad de Patologia Mamaria, Servicio de Radiodiagnóstico, Departamento de Radiología, Hospital Nacional Edgardo Rebagliati Martins. Lima, Perú. \\ ${ }^{a}$ Médico con especialidad en radiología, ORCID: https://orcid.org/0000-0002-4924-4976
}

\section{Correspondencia:}

Silvia Lucía Mayanga Sausa

lumaysa@gmail.com

Recibido: 24 de setiembre 2019

Aceptado: 17 de noviembre 2019

Publicación en línea: 28 de diciembre 2019

Conflictos de interés: La autora declara

no tener conflictos de interés.

Fuente de financiamiento: Autofinanciado

Citar como: Mayanga S. Características clínico patológicas del cáncer de mama asociadas a la expresión del marcador celular Ki-67 en el Hospital Nacional Edgardo Rebagliati Martins, 20132017. An Fac med. 2019;80(4):427-31. DOI: https://doi.org/10.15381/anales. v80i4.16746

\section{An Fac med. 2019;80(4):427-31 / DOI: https://doi.org/10.15381/anales.v80i4.16746}

\section{Resumen}

Introducción. El cáncer de mama es la tercera causa de muerte en mujeres peruanas, siendo una enfermedad genéticamente heterogénea, no existen estudios sobre el comportamiento del marcador de proliferación celular Ki-67 en esta población. Objetivo. Analizar la asociación entre las características clínico patológicas del cáncer de mama y la expresión del marcador celular Ki-67 en el Hospital Nacional Edgardo Rebagliati Martins (HNERM), Lima-Perú. Métodos. Estudio retrospectivo, realizado con las muestras diagnósticas de 209 pacientes con cáncer de mama. Las características clínico patológicas evaluadas fueron: edad, tipo histológico, tamaño tumoral, grado histológico, invasión linfovascular, ganglio linfático axilar, estadío clínico según el TNMp, receptor estrógenos (RE), receptor de progesterona (RP), y los inmunofenotipos Her2+, triple positivo (TP) y triple negativo (TN). La expresión del marcador celular Ki-67 fue categorizado como bajo (Ki$67<20 \%$ ) y alto (Ki-67>20\%). Resultados. La expresión alta del marcador celular Ki-67 se asoció con tumores de $2 \mathrm{~cm}$, grado histológico 2 y 3 , mayor número de ganglios axilares afectados y los inmunofenotipos Her2+ y triple negativo. La expresión baja del Ki-67 $(<20 \%)$ se asoció con los tumores estrógeno y progesterona positivo. Conclusión. El marcador celular Ki-67 con expresión alta (>20\%) mostró asociación significativa con características tumorales de mal pronóstico conocidas en cáncer de mama.

Palabras clave: Cáncer de Mama; Antígeno Ki-67; Perú; Estudios Transversales; Factores de Riesgo (fuente: DeCS BIREME).

\section{Abstract}

Introduction. Breast cancer is the third cause of death in peruvian women, a genetically heterogeneous disease, there are no studies on the behavior of the Ki-67 cell proliferation marker in this population. Objective. To analyze the association between the clinical pathological characteristics of breast cancer and the expression of the Ki-67 antigen in Hospital Nacional Edgardo Rebagliati Martins (HNERM), Lima-Perú. Methods. A retrospective study conducted in diagnostic samples of 209 patients with breast cancer. The clinical pathological characteristics were: age, histological type, tumor size, histological grade, lymphovascular invasion, axillary lymph node, clinical stage (TNMp), RE, RP, Her2+, triple positive, triple negative. The Ki-67 cell marker expression was categorized as low $(\mathrm{Ki}-67<20 \%)$ and high (Ki-67>20\%). Results. High expression of the Ki-67 cell marker $(>20 \%)$ was associated with tumors of $2 \mathrm{~cm}$, histological grade 2 and 3 , greater number of axillary ganglia affected and Her2+ and triple negative inmunophenotypes. Low expression of Ki-67 $(<20 \%)$ was associated with estrogen and progesterone positive tumors. Conclusion. The Ki-67 cell marker with high expression (>20\%) shows a significant association with characteristics of poor prognosis well known in breast cancer.

Keywords: Brest Cancer; Ki-67 Antigen; Peru; Cross-Sectional Studies; Risk Factors (source: MeSH NLM). 


\section{INTRODUCCION}

En el Perú, el cáncer de mama es la tercera causa de muerte en el sexo femenino, la tasa de mortalidad anual es de 8,5 casos por 100000 habitantes y constituye la segunda causa de hospitalización por neoplasia en la seguridad social peruana ${ }^{(1)}$.

El Ki-67 es una proteína nuclear descubierta por Gerdes y colaboradores en los años 80, expresa proliferación celular porque se identifica en las células en fase activa de mitosis y no se identifica durante la fase de reposo celular ${ }^{(2)}$. La técnica más usada para determinar el índice de proliferación Ki-67 es la inmunohistoquímica, la cual se basa en las reacciones antígeno - anticuerpo específico para el Ki-67 ${ }^{(3)}$. La presencia de este antígeno se asocia con alto riesgo de recurrencia y pobre sobrevida en los pacientes con cáncer de mama temprano ${ }^{(4)}$; y se relaciona con tumores poco diferenciados, mayor tamaño tumoral, y mayor número de ganglios linfáticos comprometidos ${ }^{(5)}$.

Pese a la evidencia, no se ha determinado el punto del corte del Ki-67 para clasificar a los pacientes de alto o bajo riesgo; así, en los estudios publicados, el punto de corte es arbitrario ${ }^{(4)}$, y varía en un rango que va desde $5 \%$ al $30 \%$. Gerson y col., evaluaron la asociación entre el nivel de expresión del Ki-67 y la recurrencia y supervivencia del cáncer de mama temprano, sub dividiendo la expresión del ki-67 en 4 grupos (<5\%, 6-20\%, 21 $50 \%,>50 \%)$; y concluyendo que el $\mathrm{Ki}$ 67 y el estadio clínico son los factores pronósticos más importantes para recurrencia del cáncer de mama temprano ${ }^{(6)}$.

Adicionalmente, por ser un marcador de proliferación celular, niveles altos de Ki-67 se usan como indicador de terapia quimio adyuvante ya sea sola o complementando a la terapia hormonal. El valor de referencia del Ki-67 para el tratamiento fue establecido por el panel de expertos para el tratamiento de cáncer de mama de St. Gallen del $2013{ }^{\text {(7) }}$ definiendo los subtipos moleculares hormonales en Luminal A y Luminal B según el índice de Ki-67 sea $>14 \%$; este valor fue modificado por el grupo de expertos en el 2015 modificando el punto de corte del Ki-67 en $20 \%{ }^{(8)}$. Por otro lado, Criscitiello y col. ${ }^{(9)}$, analizaron el valor predictivo del Ki-67 en la eficacia del tratamiento adyuvante con quimioterapia en pacientes con cáncer de mama receptor estrogénico positivo (Luminal B) y nódulo positivo, observando mayor beneficio al tratamiento añadiendo quimioterapia a la hormonoterapia en los pacientes con sobre expresión del Ki-67 y nódulo positivo.

Si bien se ha demostrado que el Ki67 es un factor pronóstico y predictivo importante en el cáncer de mama y con una gran utilidad en direccionar los casos que necesitan tratamiento adyuvante, en nuestro país no se ha reportado el comportamiento de este importante marcador. Por ello, el objetivo de este estudio fue analizar la asociación entre las características clínico-patológicas del cáncer de mama y la expresión del marcador celular Ki-67 en un grupo de mujeres peruanas de la seguridad social en el Hospital Nacional Edgardo Rebagliati Martins (HNERM).

\section{MÉTODOS}

\section{Diseño del estudio}

Se realizó un estudio retrospectivo, observacional y analítico, en las muestras diagnosticas de 209 pacientes con cáncer de mama biopsiadas en la Unidad de Patología Mamaria del Servicio de Radiodiagnóstico del HNERM durante el período 2013 al 2017. La unidad de análisis fue el informe anatomo-patológico resultante de las biopsias, y se complementó la información con los archivos de imágenes y las historias clínicas.

\section{Población y muestra}

La muestra fue seleccionada según los criterios de inclusión: paciente mujer con carcinoma invasor ductal o lobulillar, tratadas con mastectomía / tumorectomía, estudio del ganglio centinela y/o vaciamiento ganglionar axilar y estudio inmunohistoquimico completo. Se excluyeron los resultados incompletos, carcinomas in situ y variantes histológicas infrecuentes, cáncer metastásico a la mama y recidiva.

\section{Variables de estudio}

Las variables analizadas fueron: edad, tipo histológico, tamaño tumoral, grado histológico $(\mathrm{GH})$, invasión linfovascular, ganglio linfático axilar, estadío clínico según el TNMp, receptor estrógenos (RE), receptor de progesterona (RP), los inmunofenotipos Her2+, triple positivo (TP) y triple negativo (TN). La expresión del marcador celular Ki-67 se categorizó en 02 grupos (según el consenso de expertos para el tratamiento del cáncer de mama, St Gallen), grupo 1: Ki-67<20\% y grupo 2: Ki-67 $\geq 20 \%$.

\section{Estudio histopatológico}

Las muestras fueron analizadas en el Servicio de Anatomía Patológica del HNERM usando tinción con hematoxilina - eosina. El grado histológico se determinó siguiendo los criterios establecidos por Bloom y Richardson modificados por Elston y el tipo histológico se clasificó según la OMS. El tamaño del tumor se determinó en milímetros.

\section{Análisis inmunohistoquímico}

Se realizó el estudio inmunohistoquímico ( $\mathrm{IHQ}$ ) en los tejidos biopsiados y conservados en parafina. Los criterios de positividad para determinación de los receptores hormonales y el porcentaje de expresión del Ki-67 se determinó según el método estandarizado de la escala visual y el score de Allred (Template for reporting results of biomarker testing of specimens from patients with carcinoma of the breast, del Colegio Americano de Patólogos). El Her2+ se definió como tinción de membrana circunferencial, que es completa en más del $10 \%$ de las células tumorales.

\section{Análisis estadístico}

La media, desviación estándar (DE), valores mínimo y máximo se calcularon para las variables cuantitativas. Las variables categóricas se describieron en frecuencias. Se realizó la prueba Chi cuadrado para el estudio de asociación de variables categóricas; mientras que la comparación de medias se analizó con la prueba $\mathrm{T}$ de student, previa demostración de normalidad. La significación estadística se estableció con un valor $p<0,05$. El procesamiento estadístico se realizó en el programa IBM SPSS Statistics for Windows, Version 24.0. (IBM Corp. Released $2016{ }^{\circledR}$ ). 


\section{Aspectos éticos}

La presente investigación utiliza datos secundarios que no representa riesgo para los pacientes pues no fue necesario entrevistarlos ni obtener sus datos personales y no se requirió la revisión o aprobación por un comité de ética.

\section{RESULTADOS}

La edad promedio de las pacientes que ingresaron al estudio fue $60,2 \pm$

Tabla 1. Caracteristicas clínico patológicas del cáncer de mama en pacientes del Hospital Nacional Edgardo Rebagliati Martins, 2013-2017

\begin{tabular}{|c|c|c|c|}
\hline Característica & Indicador & Frecuencia & Porcentaje (\%) \\
\hline \multirow[t]{6}{*}{ Edad } & Media & $60,21 \pm 11,6$ años & Rango: 34-92 \\
\hline & $<40$ años & 4 & 1,9 \\
\hline & $40-<50$ años & 41 & 19,6 \\
\hline & $50-<60$ años & 46 & 22,0 \\
\hline & $60-<70$ años & 74 & 35,4 \\
\hline & $\geq 70$ años & 44 & 21,1 \\
\hline \multicolumn{4}{|l|}{ Tipo histológico } \\
\hline & Ductal & 189 & 90,4 \\
\hline & Lobulillar & 20 & 9,6 \\
\hline \multirow[t]{4}{*}{ Tamaño del tumor (TNM) } & & $1,98 \pm 1,3 \mathrm{~cm}$ & Rango: $0,15-7,0$ \\
\hline & Menor $2 \mathrm{~cm}$ & 126 & 60,3 \\
\hline & Entre $2-5 \mathrm{~cm}$ & 74 & 35,4 \\
\hline & Mayor de $5 \mathrm{~cm}$ & 9 & 4,3 \\
\hline \multicolumn{4}{|l|}{ Grado histológico } \\
\hline & Grado 1 & 55 & 26,3 \\
\hline & Grado 2 & 108 & 51,7 \\
\hline & Grado 3 & 46 & 22,0 \\
\hline \multicolumn{4}{|l|}{ Invasión linfo- vascular } \\
\hline & Positivo & 107 & 51,2 \\
\hline & Negativo & 102 & 48,8 \\
\hline \multicolumn{4}{|l|}{ Estado del ganglio axilar } \\
\hline & Negativo & 147 & 70,3 \\
\hline & $1-3(+)$ & 39 & 18,7 \\
\hline & $4-9(+)$ & 17 & 8,1 \\
\hline & $10 a+$ & 6 & 2,9 \\
\hline \multicolumn{4}{|l|}{ Estadío TNMp } \\
\hline & Estadío IA & 107 & 51,2 \\
\hline & Estadío IIA & 48 & 23 \\
\hline & Estadío IIB & 32 & 15,3 \\
\hline & Estadío IIIA & 16 & 7,7 \\
\hline & Estadío IIIB & 2 & 1 \\
\hline & Estadío IIIC & 4 & 1,9 \\
\hline \multicolumn{4}{|l|}{ Perfil Inmunohistoquímico } \\
\hline & Luminal A & 97 & 46,4 \\
\hline & Luminal B & 62 & 29,7 \\
\hline & Her2+ & 10 & 4,8 \\
\hline & Triple (+) & 14 & 6,7 \\
\hline & Triple (- ) & 26 & 12,4 \\
\hline
\end{tabular}

mente; mientras que, la invasión linfovascular estuvo presente en $51,2 \%$ y el compromiso de ganglio axilar en un 29,7\% de los casos. Los estadios TNMp más frecuentes fueron IA y IIA con 51,2 \% y $23 \%$ respectivamente (Figura 1 ). Por otro lado, los perfiles inmunohistoquímicos hormonales, tanto Luminal A y Luminal B fueron los más frecuentes con 46,4\% y $29,7 \%$; mientras que el triple negativo fue el más frecuente de los no hormonales con 12,4\% (Figura 2).

En la clasificación por grupos, según el porcentaje de expresión del Ki-67, se obtuvo 92 casos (44\%) con $\mathrm{Ki}-67<$ de $20 \%$ y 117 casos (56\%) con Ki-67 $\geq 20 \%$. En el grupo 1 la edad promedio fue 62,1 $\pm 11,2$ años y para el grupo 2 fue 58,7 $\pm 11,8$ años. Al asociar el porcentaje de expresión del Ki-67 y las características clínicopatológicas, en el grupo 2 (Ki-67 $\geq 20 \%$ ) se observó asociación significativa con tumores más grandes con diámetro promedio $2,2 \mathrm{~cm}(p=0,005)$ (Figura 3$)$, el grado histológico 2 y $3(p<0,001)$, el número de ganglios axilares positivos ( $p=0,01)$, los inmunofenotipos Her2+ $(p=0,004)$ y triple negativo $(p=0,000)$. El grupo $1(\mathrm{Ki}<20 \%)$ se asoció con los receptores hormonales,

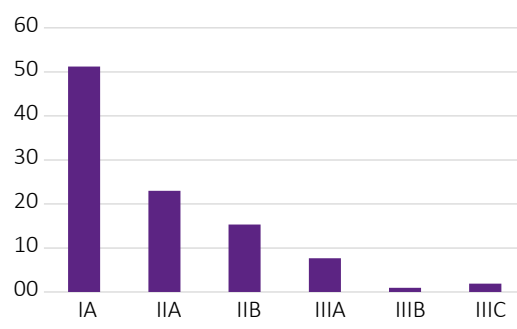

Figura 1. Estadio TNMp en pacientes con cáncer de mama en el Hospital Nacional Edgardo Rebagliati Martins, 2013-2017.

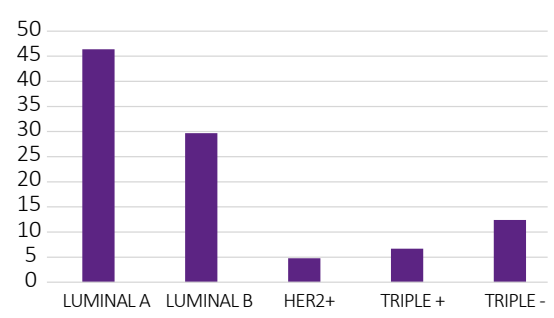

Figura 2. Perfil inmunohistoquímico en pacientes con cáncer de mama en el Hospital Nacional Edgardo Rebagliati Martins, 2013-2017. 


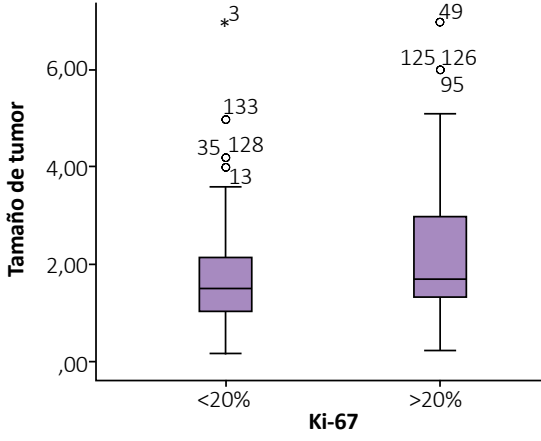

Figura 3. Comparación de medias del tamaño tumoral y la expresión del Ki-67 en pacientes con cancer de mama, en el Hospital Nacional

Edgardo Rebagliati Martins, 2013-2017.

$\operatorname{RE}$ y $R P(p=0,000$ y $p=0,001)$. No se demostró asociación estadística entre el Ki-67 y la edad, el tipo histológico y la invasión linfovascular (Tabla 2).

Tabla 2. Expresión del Ki-67 y las características clínico patológicas del cáncer de mama en pacientes del Hospital Nacional Edgardo Rebagliati Martins, 2013-2017

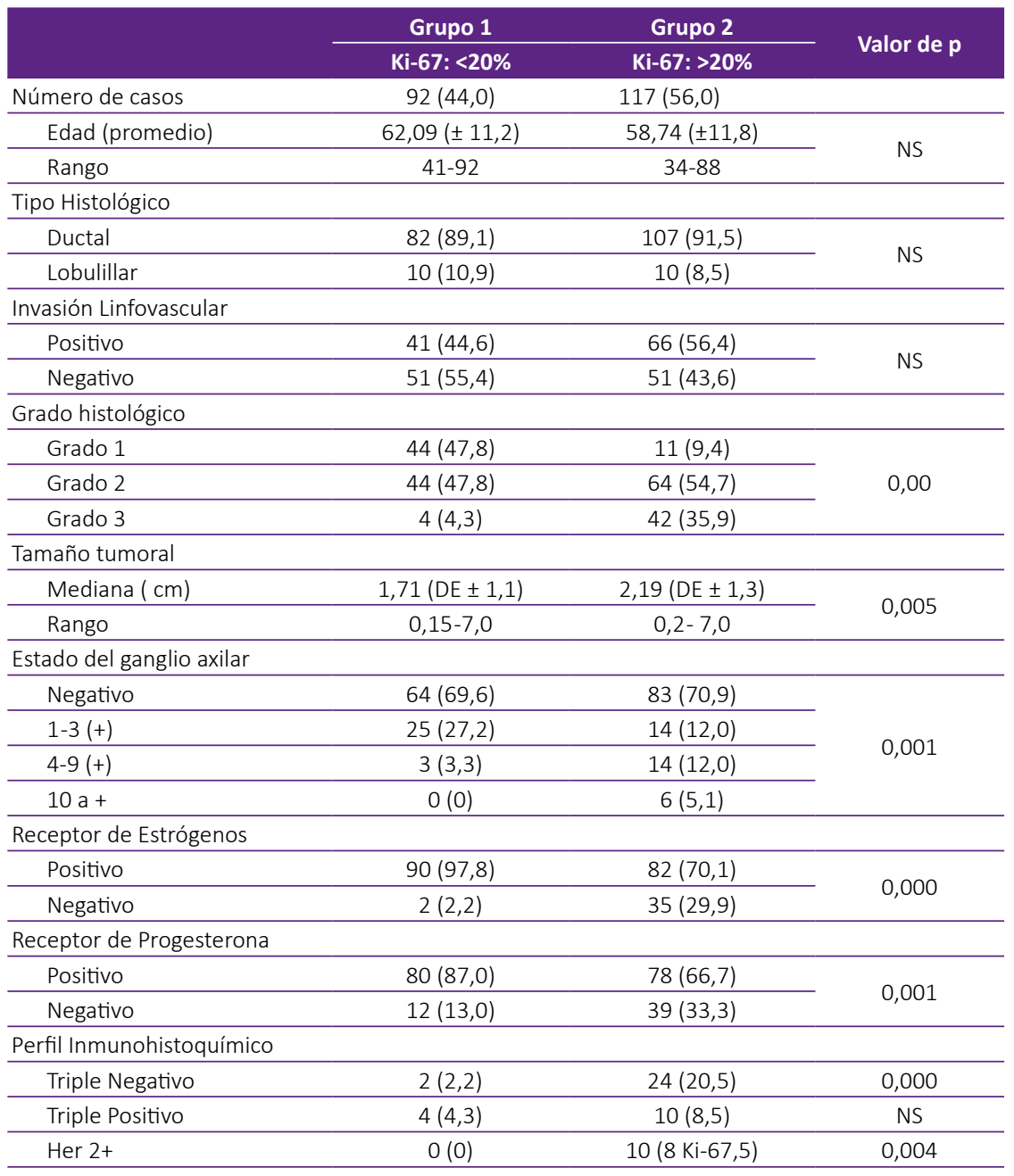

No se encontró evidencia científica previa respecto al comportamiento del marcador de proliferación celular Ki-67 en el cáncer de mama de la mujer peruana. En este estudio se encontró asociación entre alto porcentaje del Ki-67 $(\geq 20 \%)$ con el tamaño tumoral, grado histológico y afectación del ganglio axilar; lo que es similar a lo publicado por Panal y col. ${ }^{(5)}$ en una población mexicana y Ermiah y col. ${ }^{(10)}$ en mujeres libaneses, aunque en este ultimo estudio el punto de corte del Ki-67 fue de $10 \%$ y el $70 \%$ de su población fueron mujeres jóvenes menores de 50 años.

La extensión de la enfermedad hacia los ganglios axilares sigue siendo el determinante más importante de sobrevida. En nuestro estudio no fue la excepción, encontrando relación directa y significativa con el Ki-67; así, mientras mayor número de ganglios axilares comprometidos se identificaron, mayor fue el porcentaje de expresión del Ki-67 ${ }^{(11)}$.

Siendo el Ki-67 un marcador de proliferación celular que expresa mayor replicación y mitosis, se asoció a los tumores con mayor diámetro. Gerson y col. ${ }^{(6)}$, analizaron el tamaño tumoral con diferentes porcentajes de expresión del Ki-67 demostrando que el mayor tamaño tumoral se asociaba a un mayor porcentaje de expresión del Ki-67, lo que también sugería una relación con una mayor probabilidad de recurrencia.

La clasificación molecular del cáncer de mama basado en los inmunofenotipos se encuentra validada como factor pronóstico y predictivo de la enfermedad; así, el Ki-67 contribuye a la clasificación molecular del cáncer de mama y se acepta como factor predictivo para optimizar el tratamiento. Yang Hung y col. ${ }^{(12)}$ analizaron el Ki-67 como factor pronóstico en las clases moleculares de una población de 312 mujeres venezolanas, encontrando mejor pronóstico en el subgrupo Luminal A y bajo nivel Ki-67 (14,7\%); y peor pronóstico para el luminal $\mathrm{B}, \mathrm{Her} 2+\mathrm{y}$ triple negativo con valores altos de Ki-67 (25,5\%).

Según nuestros hallazgos, los tumores con RE y RP + se relacionaron significativamente con bajos niveles de Ki-67 (<20\%), lo 
que en otros estudios han llamado "relación inversa" (5); este hallazgo está en concordancia con la frecuencia de cáncer de mama inmunofenotipo Luminal A encontrada en esta población. Luporsi y col. ${ }^{(13)}$ en una revisión analítica concluyeron que el manejo de los tumores hormonales se ve favorecido cuando el Ki-67 es alto, lográndose una respuesta completa al tratamiento agregando quimioterapia, aunque tampoco se obtiene el punto de corte en el cual el Ki-67 se pueda estandarizar.

Se encontró asociación entre el inmunofenotipo triple negativo y alto porcentaje de expresión del Ki-67 ( $p=0,027)$ con valores desde $20 \%$ a más. Estas situaciones se han descrito como condiciones de peor pronóstico para recurrencia y sobrevida, también reportados por Yang Hung y col. ${ }^{(12)}$. Asimismo, Orban y col. ${ }^{(14)}$ analizaron 140 casos de cáncer de mama triple negativo encontrando que este inmunofenotipo se asoció con Ki-67 alto (promedio 70\%), además Ki-67 se comportó como un factor pronóstico dependiente de la variabilidad post neoadjuvancia.

Keam y col.(15) analizaron 105 casos de cáncer de mama triple negativo y la expresión del Ki-67 antes y después del tratamiento adyuvante, reportando una mayor probabilidad de respuesta completa al tratamiento en los casos con Ki67 alto pre tratamiento, proponiendo clasificar al cáncer de mama triple negativo en dos subtipos en base al Ki-67 con punto de corte del 10\%; sin embargo, el nivel alto de Ki-67 se asoció a peor pronóstico de sobrevida y mayor riesgo de recaída, este comportamiento paradójico de los tumores triple negativo con respecto al nivel de Ki-67 amerita mayores estudios para comprender el verdadero papel del Ki-67 en este tipo de cáncer.

En los últimos años se ha postulando al Ki-67 como indicador predictivo de padecer cáncer de mama en pacientes con enfermedad benigna de la mama e incluso se propone clasificar en alto y bajo riesgo de padecer cáncer de mama a las mujeres con diagnóstico de enfer- medad benigna y a las mujeres con genes BRCA1 y 2 alterados ${ }^{(16)}$.

La limitación de nuestro estudio fue la generación de un subregistro de pacientes con diagnóstico incompleto, en su mayoría jóvenes menores de 50 años, que sólo se realizaron la biopsia diagnóstica y no se obtuvo la pieza quirúrgica por abandono de las pacientes a la institución. Otra limitación del estudio fue que no se analizó el total de pacientes tratadas en la institución, por no tener acceso a la información de las biopsias de lesiones palpables realizadas en otro servicio.

En conclusión, en el presente estudio encontramos una asociación significativa entre el marcador de proliferación celular Ki-67 con las características tumorales de mal pronóstico en el cáncer de mama. Los resultados mostraron que los tumores de mayor tamaño, el grado histológico 2 y 3 , mayor número de ganglios linfáticos afectados y los inmunofenotipos Her2+ y triple negativo, se asociaron a alto porcentaje del Ki-67 ( $\geq 20 \%)$. Asimismo, bajos niveles de Ki-67 (<20\%) se asociaron a RE y RP+.

\section{REFERENCIAS BIBLIOGRÁFICAS}

1. Ministerio de Salud. Documento Tecnico Plan Nacional para la Prevención y Control de Cáncer de Mama en el Perú 2017-2021. 2017. Resolución Ministerial N442-2017/MINSA. [En linea]. MINSA; 2016 [citado 11 enero 2019].

2. Poblete MT. Marcadores de utilidad en cáncer mamario. Cuad Cir. 2018;15(1):74-9. DOI: 10.4206/ cuad.cir.2001.v15n1-14

3. Mannell A. The role of Ki-67 in breast cancer. South Afr J Surg Suid-Afr Tydskr Vir Chir. 2016;54(2):10-3.

4. de Azambuja E, Cardoso F, de Castro G, Colozza M, Mano MS, Durbecq V, et al. Ki-67 as prognostic marker in early breast cancer: a meta-analysis of published studies involving 12,155 patients. $\mathrm{Br}$ J Cancer. 2007;96(10):1504-13. DOI: 10.1038/ sj.bjc.6603756

5. Panal Cusati M, Herrera de la Muela M, Hardisson Hernaez D, Choqueneira Dionisio M, Román Guindo A, de Santiago Garcia FJ. Correlación entre la expresión de Ki67 con factores clásicos pronósticos y predictivos en el cáncer de mama precoz. Rev Seno Patol Mamar. 2014;27(4):163-9. DOI: 10.1016/j. senol.2014.07.005
6. Gerson-Cwilich R, Alban-de la Torre LF, VillalobosPrieto A, Padilla-Rodriguez ÁL, Serrano-Olvera JA. Recurrencia y supervivencia en cáncer de mama temprano en relación a la expresión de Ki-67. Gac Mex Oncol. 2014;13(1):4-11.

7. Untch M, Gerber B, Harbeck N, Jackisch C, Marschner N, Möbus V, et al. 13th st. Gallen international breast cancer conference 2013: primary therapy of early breast cancer evidence, controversies, consensus - opinion of a german team of experts (Zurich 2013). Breast Care Basel Switz. 2013;8(3):221-9. DOI: 10.1159/000351692

8. Coates AS, Winer EP, Goldhirsch A, Gelber RD, Gnant M, Piccart-Gebhart M, et al. Tailoring therapies--improving the management of early breast cancer: St Gallen International Expert Consensus on the Primary Therapy of Early Breast Cancer 2015. Ann Oncol Off J Eur Soc Med Oncol. 2015;26(8):1533-46. DOI: 10.1093/annonc/mdv221

9. Criscitiello C, Disalvatore D, De Laurentiis M, Gelao L, Fumagalli L, Locatelli M, et al. High Ki-67 score is indicative of a greater benefit from adjuvant chemotherapy when added to endocrine therapy in luminal B HER2 negative and node-positive breast cancer. Breast Edinb Scotl. 2014;23(1):69-75. DOI: 10.1016/j.breast.2013.11.007

10. Ermiah E, Buhmeida A, Abdalla F, Khaled BR, Salem N, Pyrhönen S, et al. Prognostic value of proliferation markers: immunohistochemical ki-67 expression and cytometric s-phase fraction of women with breast cancer in libya. J Cancer. 2012;3:421-31. DOI: 10.7150/jca.4944

11. Kermani TA, Kermani IA, Faham Z, Dolatkhah R. Ki67 status in patients with primary breast cancer and its relationship with other prognostic factors. Biomed Res Ther. 2019;6(2):2986-91. DOI: 10.15419/bmrat. v6i2.520

12. Hung CY, López L, Lizardo A, Hernández G, Saldivia F, Fernández A, et al. Expresión de KI-67 Como Factor Pronóstico en las Clases Moleculares de Carcinoma de Mama. Rev Venez Oncol. 2012;24(2):107-24.

13. Luporsi E, André F, Spyratos F, Martin P-M, Jacquemier J, Penault-Llorca F, et al. Ki-67: level of evidence and methodological considerations for its role in the clinical management of breast cancer: analytical and critical review. Breast Cancer Res Treat. 2012;132(3):895-915. DOI: 10.1007/s10549011-1837-z

14. Orban Frontini M, Ulloa Bevacqua A, Arias C, Gon C, Sanchotena V, Carrasco Maldonado M, et al. Cáncer de mama triple negativo: evaluación de características clínico-patológicas y factores pronósticos. Rev Argent Mastol. 2017;36(130):73-86.

15. Keam B, Im S-A, Lee K-H, Han S-W, Oh D-Y, Kim JH, et al. Ki-67 can be used for further classification of triple negative breast cancer into two subtypes with different response and prognosis. Breast Cancer Res BCR. 2011;13(2):R22. DOI: 10.1186/bcr2834

16. Huh SJ, Oh H, Peterson MA, Almendro V, Hu R, Bowden $\mathrm{M}$, et al. The Proliferative Activity of Mammary Epithelial Cells in Normal Tissue Predicts Breast Cancer Risk in Premenopausal Women. Cancer Res. 2016;76(7):1926-34. DOI: 10.1158/0008-5472. CAN-15-1927 